УДК 517.537

K. G. Malyutin, L. I. Studenikina

\title{
LINEAR FUNCTIONALS IN SPACE OF ENTIRE FUNCTIONS OF FINITE ORDER AND LESS THE GIVEN TYPE
}

K. G. Malyutin, L. I. Studenikina. Linear functionals in space of entire functions of finite order and less the given type, Mat. Stud. 45 (2016), 132-136.

We consider the linear topology space of entire functions of a proximate order and normal type less than the given type with respect to the proximate order. We obtain a form of continuous linear functional on this space.

1. We introduce the necessary definitions. A function $\varrho(r)$, defined on the ray $(0, \infty)$ and satisfying the Lipschitz condition on any segment $[a, b] \subset(0, \infty)$, that satisfies the conditions

$$
\lim _{r \rightarrow \infty} \varrho(r)=\rho \geq 0, \text { and } \lim _{r \rightarrow \infty} r \varrho_{+}^{\prime}(r) \ln r=0
$$

is called a proximate order.

A detailed exposition of properties of a proximate order can be found in $[1,2]$. In this paper we use the notation $V(r)=r^{\varrho(r)}$. We will assume that $V(r)$ is an increasing function on $(0, \infty)$ and $\lim _{r \rightarrow+0} V(r)=0$.

We will say that a proximate order $\varrho_{1}(r)$ is less than the proximate order $\varrho(r)$ if

$$
\lim _{r \rightarrow \infty} \varrho_{1}(r)=\rho_{1}<\rho=\lim _{r \rightarrow \infty} \varrho(r) .
$$

We now formulate a simple property of a proximate order that we shall need frequently $[1$, (2), p.33].

For $r \rightarrow \infty$ and $0<a \leq k \leq b<\infty$ the asymptotic inequality

$$
(1-\varepsilon) k^{\rho} V(r)<V(k r)<(1+\varepsilon) k^{\rho} V(r)
$$

holds uniformly in $k$.

Let $M_{f}(r)=\max _{|z|=r}|f(z)|$. If for an entire function $f(z)$ the quantity

$$
\sigma_{f}=\limsup _{r \rightarrow \infty} \frac{\log M_{f}(r)}{V(r)}
$$

is different from zero and infinity, then $\varrho(r)$ is called of a proximate order of the entire function $f(z)$ and $\sigma_{f}$ is called the type of the function $f(z)$ with respect to the proximate order $\varrho(r)$.

2010 Mathematics Subject Classification: 30D15, 30D20.

Keywords: entire function; proximate order; type; continuous linear functional. doi:10.15330/ms.45.2.132-136

(C) K. G. Malyutin, L. I. Studenikina, 2016 
Let $\varrho(r)$ be a proximate order, $\lim _{r \rightarrow \infty} \varrho(r)=\rho>0$. A single valued function $f(z)$ of the complex variable $z$ is said to belong to the space $[\varrho(r), \sigma)$ if $f(z)$ has the order less than $\varrho(r)$ or equal $\varrho(r)$ but in this second case the type is less than $\sigma$.

Thus $f \in[\varrho(r), \sigma)$ if and only if there exist $C_{f}>0$ and $\sigma_{f}>0, \sigma_{f}<\sigma$, such that $|f(z)| \leq C_{f} e^{\sigma_{f} V(|z|)}$ for all $z \in \mathbb{C}$.

A sequence of functions $\left\{f_{n}(z)\right\}$ from $[\varrho(r), \sigma)$ converges in the sense of $[\varrho(r), \sigma)$ if (i) it converges uniformly on compacts, (ii) there exists $\beta<\sigma$ such that

$$
\left|f_{n}(z)\right|<\exp [\beta V(|z|)], \quad|z|>r_{0}(\beta), n \geq 1
$$

where $r_{0}(\beta)$ does not depend on $n$.

For a suitable $C(\beta)$, which does not depend on $n$, for all $z$

$$
\left|f_{n}(z)\right|<C(\beta) \exp [\beta V(|z|)] \quad(n \geq 1) .
$$

The space $[\varrho(r), \beta)$ is a linear topology space with the sequential topology.

We introduce the function $\varphi(t)$ to be the unique solution of the equation $t=V(r)$. So

$$
\varphi(V(t))=t
$$

The following theorem on relation of the type of the function to the rate of decrease of its Taylor coefficients is true.

Theorem 1. ([1, Theorem 2', p.42]) The type $\sigma_{f}$ of an entire function $f(z)=\sum_{n=0}^{\infty} c_{n} z^{n}$ with a proximate order $\varrho(r)(\rho>0)$ is given by the equation

$$
\limsup _{n \rightarrow \infty} \varphi(n) \sqrt[n]{\left|c_{n}\right|}=\left(e \sigma_{f} \rho\right)^{1 / \rho}
$$

Let $\rho>0$. Set

$$
d_{n}=\frac{(e \sigma \rho)^{n / \rho}}{(\varphi(n))^{n}}, \quad n \geq 1, d_{0}=1 .
$$

For a function $f(z)=\sum_{n=0}^{\infty} c_{n} z^{n} \in[\varrho(r), \sigma)$, we associate the function

$$
F(z)=\sum_{n=0}^{\infty} b_{n} z^{n}, \quad b_{n}=\frac{c_{n}}{d_{n}}, n \geq 0 .
$$

It is analytic in the closed disk $|z| \leq 1$. Indeed, we have by $(4) \limsup _{n \rightarrow \infty} \varphi(n) \sqrt[n]{\left|c_{n}\right|}<(e \sigma \rho)^{1 / \rho}$, hence $\varphi(n) \sqrt[n]{\left|c_{n}\right|} \leq\left(e \sigma_{1} \rho\right)^{1 / \rho}, \sigma_{f} \sigma_{1}<\sigma, n>n_{0}$, and

$$
\left|b_{n}\right|<\left(\frac{\sigma_{1}}{\sigma}\right)^{n / \rho}, \quad n \geq n_{0} .
$$

Since $\sigma_{1}<\sigma$, we obtain

$$
\limsup _{n \rightarrow \infty} \sqrt[n]{\left|b_{n}\right|}<1
$$

and the series (5) converges in the disk $|z|<r, r>1$. 
Conversely, for any analytic function $F(z)$ in the disk $|z| \leq 1$ it corresponds the function $f(z)$ from $[\varrho(r), \sigma)$.

If a function $f(z)$ of $[\varrho(r), \sigma)$ corresponds to the function $F(z)$ as indicated above we write

$$
f(z) \sim F(z) .
$$

It is obvious, that if $f(z) \in[\varrho(r), \sigma)$ then $f(\lambda z) \sim F(\lambda z)$ in the sense of $\left[\varrho(r), \lambda^{\rho} \sigma\right)$, where $\lambda>0$ is a parameter, and if $f_{n}(z) \sim F_{n}(z)(n=1,2, \ldots, m)$ then

$$
\sum_{n=1}^{m} a_{n} f_{n}(z) \sim \sum_{n=1}^{m} a_{n} F_{n}(z) .
$$

In the present paper we prove two theorems.

Theorem 2. A sequence $\left\{f_{n}(z)\right\}$ of functions from $[\varrho(r), \sigma)$ converges in the sense of $[\varrho(r), \sigma)$ if and only if there exists $r>1$ such that the sequence $\left\{F_{n}(z)\right\}\left(f_{n}(z) \sim F_{n}(z)\right)$ converges uniformly inside the disk $|z| \leq r$ and $\left\{F_{n}(z)\right\}$ are analytic in this disk.

Theorem 3. Every continuous linear functional $l$ on the space $[\varrho(r), \sigma)$ has the form

$$
l(f)=\sum_{n=0}^{\infty} a_{n} c_{n}, \quad f(z)=\sum_{n=0}^{\infty} c_{n} z^{n},
$$

where the quantities $a_{n}$ satisfy

$$
\limsup _{n \rightarrow \infty} \varphi^{-1}(n) \sqrt[n]{\left|a_{n}\right|} \leq(e \sigma \rho)^{-1 / \rho}
$$

The case $\varrho(r) \equiv \rho>0$ was considered by A.F. Leont'ev [3, Theorem 1.1.7 and 1.1.10]. The case of the space $[\varrho(r), \sigma]$ of entire functions of type less than or equal $\sigma$ with respect to the proximate order $\varrho(r)$ was consider in [4].

2. We now prove Theorem 2. Let

$$
f_{k}(z)=\sum_{n=0}^{\infty} c_{n}^{(k)} z^{n}, \quad F_{k}(z)=\sum_{n=0}^{\infty} b_{n}^{(k)} z^{n}, k \geq 1,
$$

and let the sequence $\left\{f_{k}(z)\right\}$ converge in the sense of $[\varrho(r), \sigma)$. By Cauchy's inequality and the condition (2)

$$
\left|c_{n}^{(k)}\right|<C(\beta) \frac{\exp [\beta V(r)]}{r^{n}}, \quad|z|=r>0
$$

for some $\beta, 0<\beta<\sigma$.

Put here $r=\frac{\varphi(n)}{(\beta \rho)^{1 / \rho}}$, by (1) for and (3) we obtain

$$
V\left(\frac{\varphi(n)}{(\beta \rho)^{1 / \rho}}\right) \leq \frac{(1+\varepsilon) n}{\beta \rho}, \quad n>n_{\varepsilon} .
$$

This yields

$$
\begin{aligned}
& \left|c_{n}^{(k)}\right|<\frac{C(\beta)(\beta \rho)^{n / \rho}}{(\varphi(n))^{n}} \exp \left[\beta V\left(\frac{\varphi(n)}{(\beta \rho)^{1 / \rho}}\right)\right] \leq \\
\leq & \frac{C_{1}(\beta, \varepsilon)(\beta \rho)^{n / \rho}}{(\varphi(n))^{n}} \exp \left[\frac{(1+\varepsilon) n}{\rho}\right], n \geq 0, k \geq 1 .
\end{aligned}
$$


It follows from (2), for $z=0$,

$$
\left|c_{0}^{(k)}\right|<C_{2}(\beta), k \geq 1
$$

Therefore

$$
\left|b_{n}^{(k)}\right|=\frac{\left|c_{n}^{(k)}\right|}{d_{n}}<C_{3}(\beta, \varepsilon)\left(\frac{\beta e^{\varepsilon}}{\sigma}\right)^{n / \rho} \quad(n \geq 0, k \geq 1) .
$$

We take $r_{0}<\left(\frac{\sigma}{\beta e^{\varepsilon}}\right)^{1 / \rho}$. Since $\beta<\sigma$ we may take $\varepsilon>0$ such that $r_{0}$ is greater than 1 . For $|z|<r_{0}$

$$
\left|F_{m}(z)-F_{k}(z)\right|<\sum_{n=1}^{s}\left|b_{n}^{(m)}-b_{n}^{(k)}\right| r_{0}^{n}+2 C_{3}(\beta, \varepsilon) \sum_{n=s+1}^{\infty}\left(\frac{\beta e^{\varepsilon}}{\sigma}\right)^{n / \rho} r_{0}^{n}
$$

The series on the right-hand side converges. Given $\varepsilon_{1}$ we choose $s$ so that the second addend is less than $\varepsilon_{1}$.

Uniform convergence $\left\{f_{k}(z)\right\}$ on compacts implies that for each fixed $n$ the coefficient $c_{n}^{(k)}$ has a limit as $k \rightarrow \infty$. Then $b_{n}^{(k)}$ also has a limit as $k \rightarrow \infty$. That is why

$$
\sum_{n=1}^{s}\left|b_{n}^{(m)}-b_{n}^{(k)}\right| r_{0}^{n}<\varepsilon_{1}
$$

if $m$ and $k$ are sufficiently large. Finally, $\left|F_{m}(z)-F_{k}(z)\right|<2 \varepsilon_{1},|z|<r_{0}$.

We now prove the second part of the theorem. Let the sequence $\left\{F_{k}(z)\right\}$ converge uniformly in the disk $|z| \leq r_{0}, r_{0}>1$ and all functions are analytic in this disk. Let $f_{n}(z) \sim F_{n}(z)$. Repeating the arguments of [3] and [4], from this estimate by Theorem 1, we get that the function $\sum_{n=0}^{\infty} \frac{(\sigma e \rho)^{n / \rho}}{\left(r_{0} \varphi(n)\right)^{n}} z^{n}$ is an entire function of the proximate order $\varrho(r)$ and the type $\frac{\sigma}{r_{0}^{\rho}}$. This type is less than $\sigma$ so $r_{0}>1$. Therefore the condition (2) holds.

Since the coefficient $c_{n}^{(k)}$ has a limit as $k \rightarrow \infty$ for each fixed $n$ it is possible to prove similarly to the previous that the sequence $\left\{f_{k}(z)\right\}$ converges uniformly on compact sets. So this sequence converges in the sense of $[\varrho(r), \sigma)$.

Remark 1. If the sequence $\left\{f_{k}(z)\right\}$ converges to $f(z)$ in the sense of $[\varrho(r), \sigma)$, and $\left\{F_{k}(z)\right\}$ converges to $F(z)$ then $f(z) \sim F(z)$.

3. By Theorem 2 it can be argued that the space $[\varrho(r), \sigma)$ of functions $f(z)$ with convergence in the sense of $[\varrho(r), \sigma)$ is transform onto the space $A(\bar{D})$ of functions $F(z)$ analytic in the disk $\bar{D}=\{z:|z| \leq 1\}$. A sequence $\left\{F_{k}(z)\right\}$ of functions from $A(\bar{D})$ converges to $F(z)$ in the sense of $A(\bar{D})$ if there exists $r_{0}>1$ such that all $\left\{F_{k}(z)\right\}$ are analytic in the disk $D_{0}=\left\{z:|z| \leq r_{0}\right\}$ and $\left\{F_{k}(z)\right\}$ converges to $F(z)$ by the norm $\|F\|=\max _{z \in \bar{D}_{0}}|F(z)|$.

Next, using the form of a linear functional in the space $[\varrho(r), \sigma]([4])$, and the arguments of the proof of Theorem 1.1.10 [3], it is not difficult to establish the validity of Theorem 3 .

We recall the following known facts $([3$, p. 17-18]):

1) Let $F_{n}(z) \in A(\bar{D})(n \geq 1)$. In order to a function $F_{0}(z) \in A(\bar{D})$ can be approximated with an arbitrary accuracy by linear combinations of functions $F_{n}(z)$ it is necessary and sufficient that the equalities

$$
L\left(F_{n}\right)=0, \quad n \geq 1,
$$


where $L(F)$ is an arbitrary continuous linear functional on $A(\bar{D})$, imply $L\left(F_{0}\right)=0$. In particular, the system of functions $\left\{F_{n}(z)\right\}$ is complete in $A(\bar{D})$ if and only if equalities (8) imply that for arbitrary $L$ one has $L(F)=0$ for any function $F(z) \in A(\bar{D})$.

2 ) Let $M$ be a proper closed set in $A(\bar{D})$ and $F_{0} \in A(\bar{D})$. Then there exists a functional $L(F)$ with the property: $L(F)=0$ for all $F \in M$ but $L\left(F_{0}\right) \neq 0$.

Note that a closed set $M$ in $A(\bar{D})$, corresponds to a closed set in the space $[\varrho(r), \sigma)$ by the relation $f(z) \sim F(z)$.

In virtue of these facts we obtain the following statements:

1) Let $f_{n}(z) \in[\varrho(r), \sigma)(n \geq 1)$. In order to a function $f_{0}(z) \in[\varrho(r), \sigma)$ can be approximated with arbitrary accuracy by linear combinations of functions $f_{n}(z)$ (in the sense of $[\rho(r), \sigma))$ it is necessary and sufficient that equalities

$$
l\left(f_{n}\right)=0 \quad(n \geq 1),
$$

where $l(f)$ is an arbitrary continuous linear functional on $[\varrho(r), \sigma)$, imply $l\left(f_{0}\right)=0$. In particular, the system of functions $\left\{f_{n}(z)\right\}$ is complete in $[\varrho(r), \sigma)$ if and only if equalities (9) imply $l(f)=0$ for any function $f(z) \in[\varrho(r), \sigma)$.

$2)$ Let $N$ be a closed set in $[\varrho(r), \sigma)$ that does not coincide with the $[\varrho(r), \sigma)$ and $f_{0}(z) \in$ $[\varrho(r), \sigma) \backslash N$. Then there exists a functional $l(f)$ with the property: $l(f)=0$ for all $f(z) \in N$ but $l\left(f_{0}\right) \neq 0$.

\section{REFERENCES}

1. B.Ya. Levin, Distribution of zeros of entire functions, English revised edition Amer. Math. Soc, Providence, RI, 1980.

2. N.H. Bingham, C.M. Goldie, J.L. Teugels, Regular variation, Cambridge university press, Cambridge, London, New-York, New Rochele, Melburn, Sydney, 1987.

3. A.F. Leont'ev, Obobshcheniye ryadov e'ksponent, Nauka, 1981. (in Russian)

4. K.G. Malyutin, T.I. Malyutina, Linear functionals in some spaces of entire functions of finite order, Istanbul University Science Faculty the Journal of Mathematics, Physics and Astronomy, 6 (2015), 1-6.

Southwest State University

Kursk, Russia

malyutinkg@gmail.com 